\title{
Simultaneous Bedside Assessment of Global Cerebral Blood Flow and Effective Cerebral Perfusion Pressure in Patients with Intracranial Hypertension
}

\author{
M. Jägersberg $\cdot$ C. Schaller $\cdot$ J. Boström • \\ B. Schatlo $\cdot$ M. Kotowski $\cdot$ C. Thees
}

Published online: 25 February 2010

(C) Humana Press Inc. 2010

\begin{abstract}
Background We examined a bedside technique transcerebral double-indicator dilution (TCID) for global cerebral blood flow (CBF) as well as the concept of effective cerebral perfusion pressure $\left(\mathrm{CPP}_{\text {eff }}\right)$ during different treatment options for intracranial hypertension, and compared global $\mathrm{CBF}$ and $\mathrm{CPP}_{\text {eff }}$ with simultaneously obtained conventional parameters.

Methods Twenty-six patients developing intracranial hypertension in the course of traumatic brain injury or subarachnoid hemorrhage were prospectively analyzed using a combined assessment during elevated ventilation $(n=15)$ or osmotherapy (hypertonic saline or mannitol). For calculation of global CBF, injections of ice-cold indocyanine green boluses were performed and temperature and dye concentration changes were monitored in the thoracic aorta and the jugular bulb. CBF was then calculated according to the mean transit time principle. Estimation of CCP, the arterial pressure at which cerebral blood flow becomes zero, was performed by synchronized registration of corresponding values of blood flow velocity in the middle cerebral artery and arterial pressure and extrapolation to zero-flow velocity. $\mathrm{CPP}_{\text {eff }}$ was
\end{abstract}

M. Jägersberg $(\bowtie) \cdot$ C. Schaller · B. Schatlo · M. Kotowski Department of Neurosurgery, University of Geneva Medical Center, Geneva, Switzerland

e-mail: max.jaegersberg@bluewin.ch

M. Jägersberg · C. Schaller · J. Boström · B. Schatlo · M. Kotowski

Department of Neurosurgery, University of Bonn Medical Center, Bonn, Germany

C. Thees

Department of Anaesthesiology, University of Bonn Medical Center, Bonn, Germany calculated as mean arterial pressure minus critical closing pressure $\left(\mathrm{CPP}_{\text {eff }}=\mathrm{MAP}_{\mathrm{c}}-\mathrm{CCP}\right)$.

Results Elevated ventilation causes a decrease in both ICP $(P<0.001)$ and CBF $(P<0.001)$. While $\mathrm{CPP}_{\text {conv }}$ increased $(P<0.001), \mathrm{CPP}_{\text {eff }}$ decreased during this observation $(P=0.002)$. Administration of osmotherapeutic agents resulted in a decrease of ICP $(P<0.001)$ and a temporary increase of $\mathrm{CBF}(P=0.052) . \mathrm{CPP}_{\text {conv }}$ and $\mathrm{CPP}_{\text {eff }}$ showed no striking difference under osmotherapy.

Conclusion TCID allows repeated measurements of global CBF at the bedside. Elevated ventilation lowered and osmotherapy temporarily raised global CBF. In situations of increased vasotonus, $\mathrm{CPP}_{\text {eff }}$ is a better indicator of blood flow changes than conventional $\mathrm{CPP}$.

Keywords Transcerebral double-indicator dilution technique - Global cerebral blood flow - Effective cerebral perfusion pressure $\cdot$ Critical closing pressure $\cdot$ Intracranial hypertension · Mannitol · Hypertonic saline

\section{Introduction}

Insufficient cerebral blood flow (CBF) is associated with poor outcome in patients suffering from intracranial hypertension higher than $20 \mathrm{mmHg}$. Therefore, it is a general practice in neurointensive care units to monitor and assure a correct cerebral perfusion pressure (CPP) to ensure sufficient blood flow in these patients [1]. Conventionally, $\mathrm{CPP}$ is calculated as cerebral mean arterial blood pressure $\left(\mathrm{MAP}_{\mathrm{c}}\right)$ minus intracranial pressure $(\mathrm{ICP})\left(\mathrm{CPP}_{\text {conv }}=\right.$ $\left.\mathrm{MAP}_{c}-\mathrm{ICP}\right)$. Therapeutic interventions such as elevated ventilation or administration of osmotic agents in patients with intracranial hypertension are recommended to reduce ICP and in turn increase CPP [1]. However, this equation 
does not include all factors that affect CPP, e.g., vasomotor tone and venous pressure [2]. From a physiological point of view, the effective CPP is the difference between momentary arterial pressure and the pressure at which cerebral blood flow in the precapillary bed suspends [3]. Determining this particular pressure, called critical closing pressure $(\mathrm{CCP})\left(\mathrm{CPP}_{\text {eff }}=\mathrm{MAP}_{\mathrm{c}}-\mathrm{CCP}\right)[3-5]$ and obtainable with help of transcranial Doppler, can be used to monitor CPP.

In addition to mere observation of perfusion pressure (CPP) there is a strong interest in direct measurement of perfusion itself (CBF). Invasive probes, however, can only indicate regional blood flow conditions, and patient transfer from the neurointensive care unit to radiological units to assess global CBF provides only momentary values and presents a high risk for critically unstable patients. In order to overcome these disadvantages a new method has been introduced for the bedside monitoring of global CBF in intensive care patients that refers to the mean transit time principle [6], the transcerebral double-indicator dilution technique (TCID) which has shown a good correlation of $\mathrm{CBF}$ values in comparison to the Kety-Schmidt method, as well as good reliability [7-10].

In this study, we assessed global CBF by means of the TCID technique and calculated $\mathrm{CPP}_{\text {eff }}$ and $\mathrm{CPP}_{\text {conv }}$ in patients with intracranial hypertension in the course of two medical therapy options, elevated mechanical ventilation or osmotherapy. Our aim is to test the two methods for determination of global CBF and $\mathrm{CPP}_{\text {eff }}$ in clinical practice and to find out whether $\mathrm{CPP}_{\text {eff }}$ could be superior to $\mathrm{CPP}_{\text {conv }}$ in indicating cerebral blood flow changes.

\section{Methods}

Patient Characteristics and Intensive Care Management

This prospective clinical study was approved by the University of Bonn Institutional Review Board and written consent was obtained from all the patients or their closest relatives.

All the patients underwent simultaneous bedside assessment of global CBF, ICP, CPP ${ }_{\text {conv }}, \mathrm{CCP}$, and $\mathrm{CPP}_{\text {eff }}$ together with basic hemodynamic parameters such as heart rate (HR), continuous cerebral arterial pressure $\left(\mathrm{AP}_{\mathrm{c}}\right)$, mean cerebral arterial pressure $\left(\mathrm{MAP}_{\mathrm{c}}\right)$, and arterial and cerebrovenous blood gas samples. All the patients were kept under sedation, analgesia, and mechanical ventilation according to the local intensive care unit regimen for patients with intracranial hypertension.

\section{Patient Groups}

Patients admitted to the University of Bonn Medical Center are addressed either to the Anaesthesiology or to the
Neurosurgery intensive care unit, depending on the severity of the brain lesions.

\section{Group 1 (Elevated Ventilation)}

Group 1 included 15 consecutive patients treated in the intensive care unit of the Department of Anaesthesiology, for initially mild traumatic brain injuries (MTBI). Traumatic CT findings were present in all these patients. The injuries were judged mild due to clinical impact and radiologic extension and were graded according to the initial Marshall CT Sore [11, 12]. The patients developed intracranial hypertension during their hospitalization in the course of systemic inflammatory response syndromes (SIRS) $[13,14]$ and were treated by an elevation of mechanical ventilation in reference to their baseline settings to lower arterial carbon dioxide tension $\left(\mathrm{PaCO}_{2}\right)$ by $7 \mathrm{mmHg}$. Measurements were performed under baseline ventilation conditions $\left(T_{0}\right)$ and after undergoing elevated ventilation for approximately $30 \mathrm{~min}\left(T_{\text {ElevVent }}\right)$ (Fig. 1).

\section{Group 2 (Osmotherapy)}

Group 2 included a consecutive group of 11 patients with intracranial hypertension due to aneurysmal subarachnoid hemorrhage (SAH) or severe traumatic brain injury (TBI), hospitalized in the Department of Neurosurgery. As for Group 1, the Marshall CT Score was applied for TBI patients and the WFNS Scale for SAH patients $[11,12,15]$. Patients in Group 2 were treated by intravenous administration of the hypertonic saline HyperHES ${ }^{\circledR} 7.2 \%(n=8)$ or, if baseline sodium serum levels were too high, by mannitol 20\% $(n=3)$. As in group 1, the baseline measurement $T_{0}$ was performed immediately prior to therapy. Then, the respective infusions of $150 \mathrm{mg} / \mathrm{kg} \mathrm{b.w}$. as for HyperHES $^{\circledR}$ and $0.5 \mathrm{~g} / \mathrm{kg}$ b.w. as for mannitol (=usual amount for a single adult application), were administered within $15 \mathrm{~min}$. Measurements were repeated $15\left(T_{15}\right)$ and $60 \min \left(T_{60}\right)$ after administration (Fig. 1).

\section{Measurement of Global CBF via TCID Technique}

CBF was measured with the TCID technique [10]. The underlying theory to this method is the mean transit time principle according to which blood flow through the brain can be calculated when the transcerebral mean transit time of a diffusible indicator from the organ's arterial inlet to its venous outlet as well as the partition coefficient $\lambda$ between brain and blood for the respective indicator are known $(\mathrm{CBF}=\lambda / \mathrm{mtt} \quad[\mathrm{ml} / \mathrm{s} / \mathrm{g}])[16]$. The transcerebral mean transit time in turn consists of an intravascular and an extravascular part $\left(\mathrm{mtt}=\mathrm{mtt}_{\mathrm{iv}}+\mathrm{mtt}_{\mathrm{ev}}\right)$ [17, 18]. The TCID technique makes use of two simultaneously 


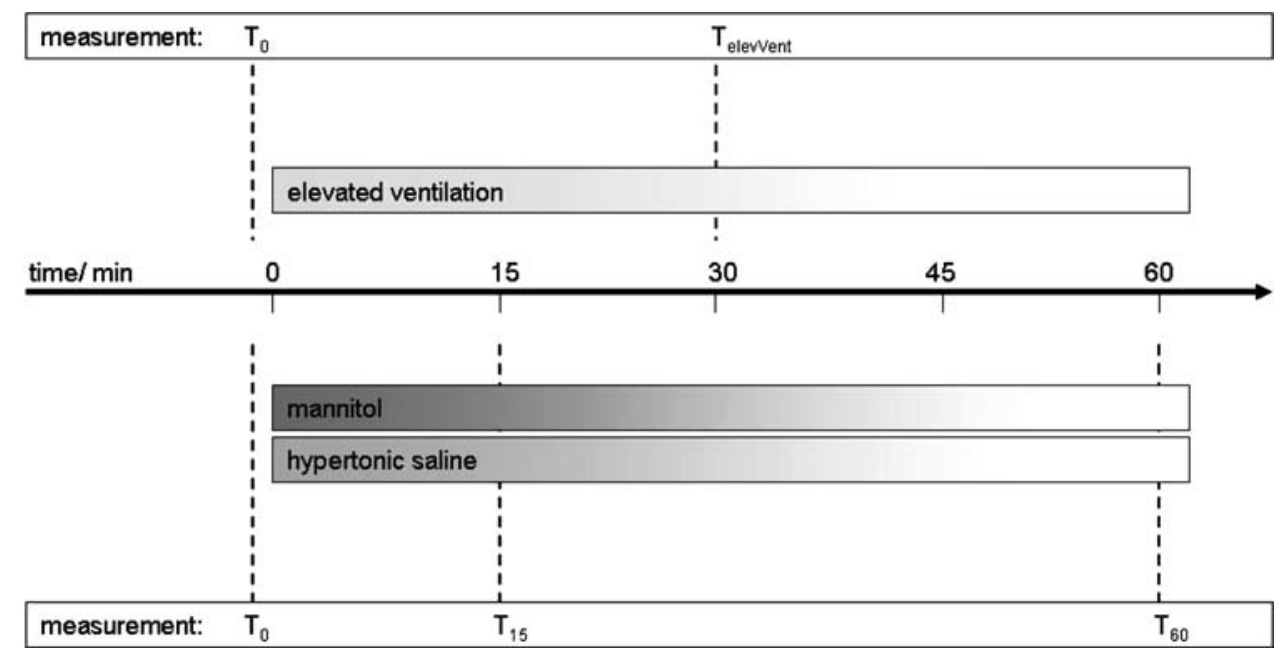

Fig. 1 Study protocol for patients undergoing elevated ventilation (Group 1) or osmotherapy (Group 2). Time points of measures: $T_{0}=$ prior to elevated ventilation or prior to osmotherapy, respectively; $T_{\text {ElevVent }}=$ under elevated ventilation of approximately $30 \mathrm{~min} ; T_{15}=15 \mathrm{~min}$ after onset of osmotherapy (hypertonic saline

administered indicators: negative heat (ice-cold isotonic saline bolus injections), serving as a highly diffusible indicator, and dye concentration (indocyanine green in the bolus liquid) serving as a strictly intravascular indicator. If after indicator injection their concentration changes over time at the arterial inlet and venous outlet of the brain are continuously recorded, then the mean transit time can be calculated using computer-assisted via a transport function [16]. Negative heat is sufficient for calculation of CBF, but indocyanine green in addition allows to verify the intravascular part of the transport function [17-19]. The partition coefficient for the highly diffusible heat can be assumed to be $1 \mathrm{ml} / \mathrm{g}[20](\mathrm{CBF}=1 / \mathrm{mtt}[\mathrm{ml} / \mathrm{g} / \mathrm{s}])$ and the formula can be modified to the standard unit for CBF $(\mathrm{CBF}=60 \times 100 / \mathrm{mtt}[\mathrm{ml} / 100 \mathrm{~g} / \mathrm{min}])[10]$.

Technically, the method requires simultaneous registration of thermo- and dye-dilution curves via two intravascular fiberoptic thermistor catheters at the arterial inflow and venous outflow of the brain as well as a central venous line for the indicator injection (Fig. 2). One catheter (PV-2024, 4 French, Pulsion Medical Devices, Munich, Germany) was placed in the thoracic aorta via retrograde puncture of the left femoral artery. The second catheter was placed in the jugular bulb via retrograde puncture of the internal jugular vein. Correct positioning of the catheter tips was verified by fluoroscopy. Rapid bolus injections of ice-cold indocyanine green (standard amount $25 \mathrm{mg}$ in $40 \mathrm{ml} \mathrm{NaCl}, T<5^{\circ} \mathrm{C}$ ) into the central venous line were performed and the resulting inlet and outlet dilution curves for the two indicators were digitally recorded (COLD Z-021, Pulsion, Munich, Germany, or mannitol); $T_{60}=60 \mathrm{~min}$ after onset of osmotherapy. Measurements contained global $\mathrm{CBF}, \mathrm{ICP}, \mathrm{AP}_{\mathrm{c}}, \mathrm{MAP}_{\mathrm{c}}, V_{\mathrm{MCA}}$, arterious, and cerebrovenous blood gas samples. $\mathrm{CCP}, \mathrm{CPP}_{\text {eff }}$, and $\mathrm{CPP}_{\text {conv }}$ were calculated from these parameters

Fig. 2). CBF was then calculated offline with a portable computer by curve analysis software written in Pascal [10].

Arterial and Intracranial Pressure Measurements

Cerebral arterial pressure was continuously monitored and digitally recorded at a sample rate of $50 \mathrm{~Hz}$ (for assessment of CPP, see below) by the same intravascular catheter located in the thoracic aorta, with a casual pressure transducer calibrated to the level of the Foramen of Monro. Mean arterial pressure $\left(\mathrm{MAP}_{\mathrm{c}}\right)$ was calculated from these recordings. ICP was monitored by means of conventional intraventricular probes (Duisburger Nadel; Pilling Weck, Karlstein, Germany).

Critical Closing Pressure and $\mathrm{CPP}_{\text {eff }}$

Estimation of critical closing pressure (CCP) is based on the fact that the regression of cerebral AP of each single heart beat and corresponding flow velocity is linear and, when extrapolated, shows a positive pressure intercept at zero-flow velocity [2]. At this arterial pressure, in the small cerebral resistance vessels the effective downstream pressure becomes zero and vessel flow suspends, consequently causing zero-flow velocity at the arterial observation site, in our study the middle cerebral artery. Continuous monitoring of flow velocity in the middle cerebral artery $\left(V_{\mathrm{MCA}}\right)$ was performed at a depth of $45-55 \mathrm{~mm}$ with a $2-\mathrm{MHz}$ transcranial Doppler probe (Multidop T; DWL, Sipplingen, Germany) fixed to the patient's head using a deviceadapted holder apparatus. All Doppler probe adjustments 


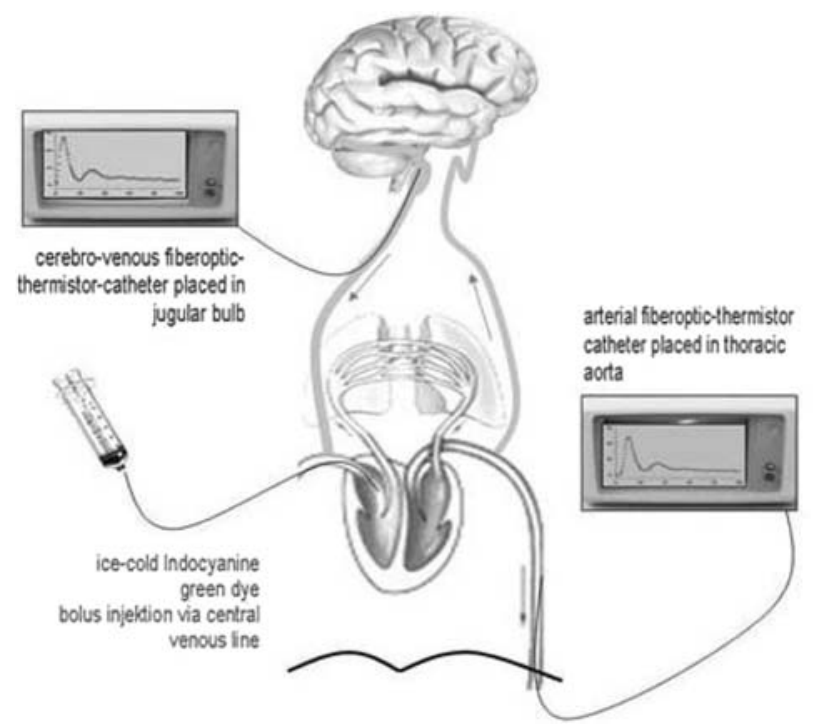

Fig. 2 Transcerebral double-indicator dilution technique. Left: icecold indocyanine green dye bolus injections into the right atrium were performed. Two combined fiberoptic thermistor catheters placed in the thoracic aorta and the jugular bulb registered resulting thermoand dye-dilution curves at the respective sites. Right: typical thermo$(\Delta T)$ and dye-dilution $\left(C_{\mathrm{ICG}}\right)$ changes over time measured at arterial

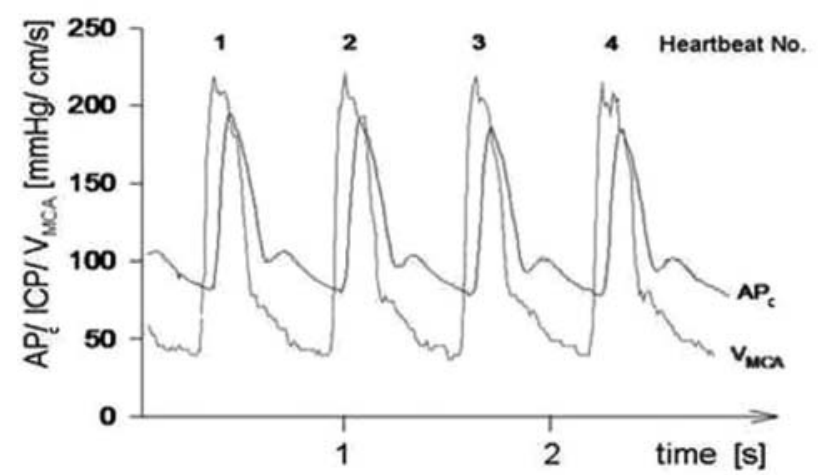

Fig. 3 Estimation of the critical closing pressure for determination of effective cerebral perfusion pressure. Left: graph of simultaneously recorded parameters of $\mathrm{AP}_{\mathrm{c}}$ calibrated to the level of the head and $V_{\mathrm{MCA}}$ in the middle cerebral artery drawn over time at a sample rate of $50 \mathrm{~Hz}$. Corresponding values of pressure and flow velocity were found shifting the curves to maximal correlation using iterative

were performed by the same investigator (M. Jägersberg). No patient suffered angiographic vasospasm at the moment of assessment. $\mathrm{AP}_{\mathrm{c}}$ was monitored in the aorta as mentioned above. Both curves of $\mathrm{AP}_{\mathrm{c}}$ and $V_{\mathrm{MCA}}$ were simultaneously recorded at a sample rate of $50 \mathrm{~Hz}$ (Fig. 3). Offline, the time lag between the two curves was compensated by iterative regression analysis to find corresponding pressure-flow values and CCP was estimated in a heartbeat-to-heartbeat analysis from zero-flow velocity as extrapolated by digital regression analysis of

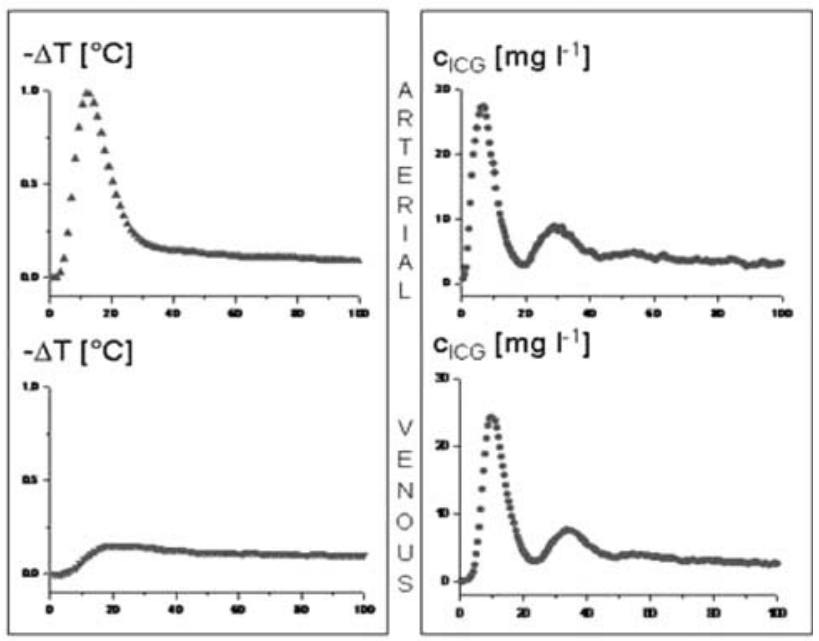

inlet (aorta) and venous outlet (jugular bulb) of the brain. Differences in delay and damping of the jugular curves between the two indicators are due to the kinetic character of the respective indicator. With help of these arterial inlet and venous outlet curves immediate calculation of global CBF was possible (based on [10])

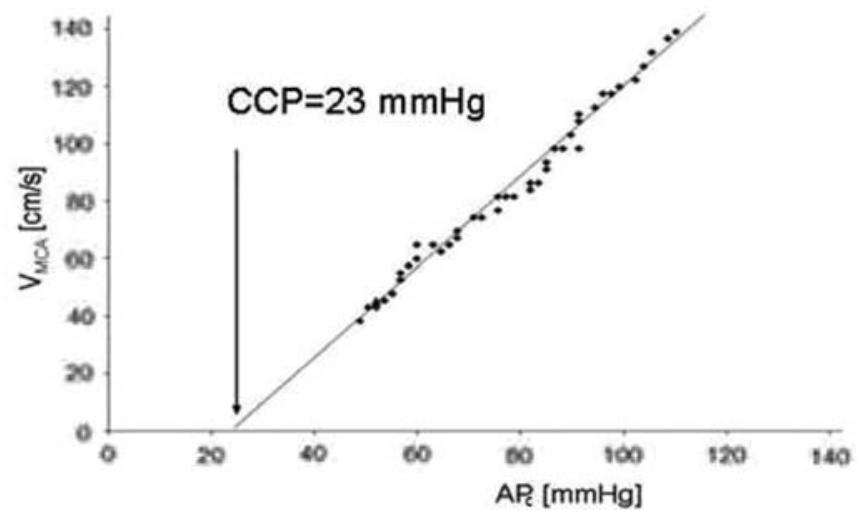

regression analysis. Right: values of $V_{\mathrm{MCA}}$ drawn over corresponding values of $\mathrm{AP}_{\mathrm{c}}$ for a single heartbeat. Determination of CCP by a regression line extrapolated to zero-flow velocity. CCPs for each single heartbeat were obtained over $30 \mathrm{~s}$ and afterward averaged to rule out respiration artifacts (based on [2])

$\mathrm{AP}_{\mathrm{c}}-V_{\mathrm{MCA}}$ plots (Fig. 3). During $30 \mathrm{~s}$, CCPs of each heartbeat were estimated and then averaged to gain an actual CCP independent of respiration artifacts. Hence $\mathrm{CPP}_{\text {eff }}$ was calculated as $\mathrm{MAP}_{\mathrm{c}}$ minus CCP [2].

\section{Statistics}

Data in figures and text are expressed as mean \pm standard deviation (SD), in absolute numbers and relative percentage changes to corresponding baseline levels $\left(T_{0}=100 \%\right)$ 
for each treatment. Correlation and significance using the Pearson's correlation coefficient $(r)$ and Student's $t$ test, respectively, was calculated with help of Microsoft Excel ${ }^{\circledR}$ 2003 SP3.

\section{Results}

In total, 63 measurements for $\mathrm{CBF}, \mathrm{CPP}_{\text {conv }}$ and $\mathrm{CPP}_{\text {eff }}$ were obtained in 26 patients during hyperventilation [Group $1, n=15$, aged $54 \pm 17$ years, 10 men, 5 women; MTBI with initial Marshall CT Scores $2(\times 4), 3(\times 7)$ and 6 $(\times 4)$ ] and during osmotherapy [Group 2, $n=11$, aged $53 \pm 18$ years, 6 men, 5 women; SAH $n=6$, WFNS Grades 4 and 5, TBI $n=5$, Marshall CT Score $3(\times 1)$, $4(\times 2)$ and $5(\times 2)$ ]. Relevant results are shown in Fig. 4. No complications were observed in relation to catheterization, indicator injection, or the therapy applied.

\section{Technical Aspects of the TCID Technique}

Preparation of the indicator injections took about $5 \mathrm{~min}$ and had to be performed $30 \mathrm{~min}$ prior to measurement to ensure ice-cold temperature. Retrograde puncture of the internal jugular vein was only performed by trained intensivists. Due to procedure-inherent problems (mainly insufficient indicator injection speed or catheter tip contact with the vessel walls) $28.6 \%$ of all measurements had to be repeated. Average injection volume and dye dose per patient was $102.9 \mathrm{ml}$ and $64.3 \mathrm{mg}$ for patients undergoing elevated ventilation, $154.3 \mathrm{ml}$ and $96.4 \mathrm{mg}$ for patients treated with osmotherapy.

\section{Elevated Ventilation}

The $\mathrm{PaCO}_{2}$ drop in group 1 during elevated ventilation $\left(\mathrm{PaCO}_{2}\right.$ at $T_{0} 43.7 \pm 3.3 \mathrm{mmHg} ; \mathrm{PaCO}_{2}$ at $T_{\text {ElevVent }}$ $36.4 \pm 4.2 \mathrm{mmHg}, \quad P<0.001)$ was associated with a significant decrease in ICP $(P<0.001)$ and in $\mathrm{CBF}$ $(P<0.001)$ in comparison to baseline ventilation levels. Systemic parameters as HR and MAP remained unchanged. The resulting increase in $\mathrm{CPP}_{\text {conv }}$ contrasts the decrease of $\mathrm{CPP}_{\text {eff }}$ obtained in the same patients. Correlation between $\mathrm{CPP}_{\text {conv }}$ and CBF was -0.29 , between $\mathrm{CPP}_{\mathrm{eff}}$ and CBF 0.35 without reaching significance (Fig. 4).

\section{Osmotherapy}

Results of hypertonic saline and mannitol are presented separately due to their different time effects concerning onset and duration.

In the eight patients treated with hypertonic saline a significant decrease in ICP $(P<0.0001)$ during the first
$15 \min \left(T_{0}-\mathrm{T}_{15}\right)$ was observed. The further decrease during the next 45 min remained significant $(P<0.001)$ compared to baseline ICP $\left(\mathrm{T}_{0}-\mathrm{T}_{60}\right)$. CBF increased significantly after $15 \mathrm{~min}(P=0.024)$. However, this effect was no longer present after $60 \mathrm{~min}$ with flow values of $\mathrm{CBF}$ comparable to baseline level. Both, $\mathrm{CPP}_{\text {conv }}$ and $\mathrm{CPP}_{\text {eff }}$ had similar trends without statistical significance $\left(r\left[\mathrm{CPP}_{\text {conv }}-\right.\right.$ $\left.\mathrm{CBF}]=0.3 ; \mathrm{r}\left[\mathrm{CPP}_{\mathrm{eff}}-\mathrm{CBF}\right]=0.59\right)($ Fig. 4).

As for the three patients treated with mannitol, a strong decrease in ICP was accompanied by an important, observation period lasting $\left(T_{0}-T_{60}\right)$ increase in $\mathrm{CBF}$. $\mathrm{CPP}_{\text {conv }}$ and $\mathrm{CPP}_{\text {eff }}$ had similar trends to the corresponding $\mathrm{CBF}$ $\left(r\left[\mathrm{CPP}_{\mathrm{conv}}-\mathrm{CBF}\right]=-0.42 ; r\left[\mathrm{CPP}_{\mathrm{eff}}-\mathrm{CBF}\right]=-0.03\right)$ (Fig. 4). Due to the small number of patients in this subgroup none of the observations reached significance.

\section{Discussion}

In this clinical study, we investigated global $\mathrm{CBF}$ at the bedside with the TCID technique in patients with intracranial hypertension in the course of ICP reduction by either elevated ventilation or osmotherapy and evaluated the correlation of global CBF with simultaneously obtained

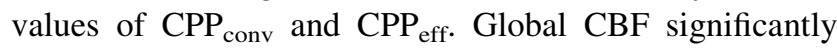
decreased under elevated ventilation and increased under osmotherapy. During elevated ventilation, $\mathrm{CPP}_{\text {eff }}$ was superior when compared with conventially obtained CPP in reflecting global $\mathrm{CBF}$ changes, while after osmotherapy there was no advantage in assessing $\mathrm{CPP}_{\text {eff }}$ when compared with $\mathrm{CPP}_{\text {conv. }}$ A possible explanation is the strong vasotonus following hypocapnia that is not present to this extent during osmotherapy.

\section{Intracranial Hypertension}

The pathologies included in the study, all possibly leading to intracranial hypertension, were MTBI, TBI and SAH. As for SAH and TBI, pathophysiologic pathways have been well examined and we refer to the literature for details [2126]. Patients with initially MTBI with minor structural CT findings may suffer from delayed intracranial hypertension in the course of a SIRS [13]. The pathogenesis of delayed intracranial hypertension as a "second hit" in these patients is not completely understood. The systemic inflammatory response primarily affects organs other than the bloodbrain-barrier-protected central nervous system. However, initial mild brain injury weakening this barrier may expose the brain tissue to the inflammatory process and thus vasogenic and cellular edema [13, 14].

The initial ictus of the diseases mentioned above is different. However, in clinical practice, patients suffering from intracranial hypertension are all treated similarly 
Fig. 4 Results of monitored parameters for patients undergoing elevated ventilation (Group 1) or osmotherapy (Group 2, results for hypertonic saline and mannitol presented separately) in mean \pm standard deviation (SD) for absolute numbers and percentage changes to baseline levels $\left(T_{0}=100 \%\right)$. Histogram showing parameter changes in percent. $P$ values calculated with Student's $t$ test. $H R$ heart rate, $M A P_{c}$ mean arterial pressure calibrated with Foramen of Monro, $C I$ cardiac index, $C B F$ global cerebral blood flow, ICP intracranial pressure, $C P P_{\text {conv }} / C P P_{\text {eff }}$ conventional/effective cerebral perfusion pressure, $S_{j v}$ jugular venous oxygen saturation, $N S$ not statistically significant

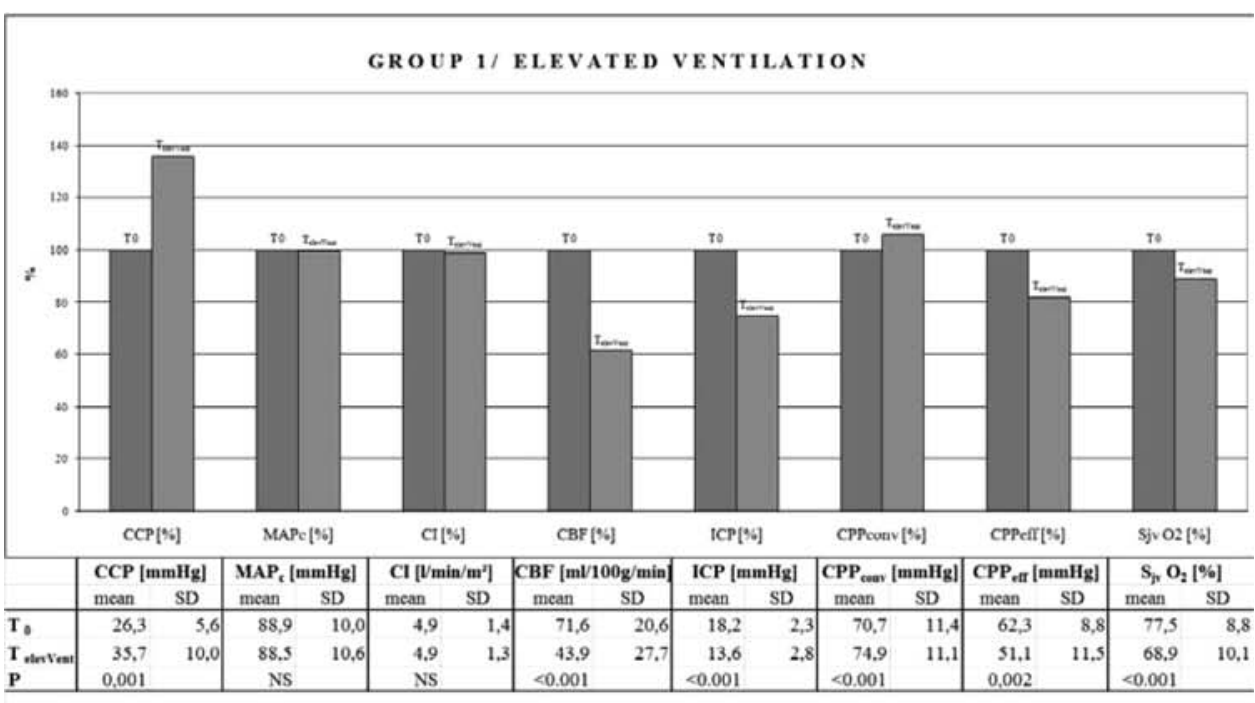

GROUP $2 /$ HYPERTONIC SALINE

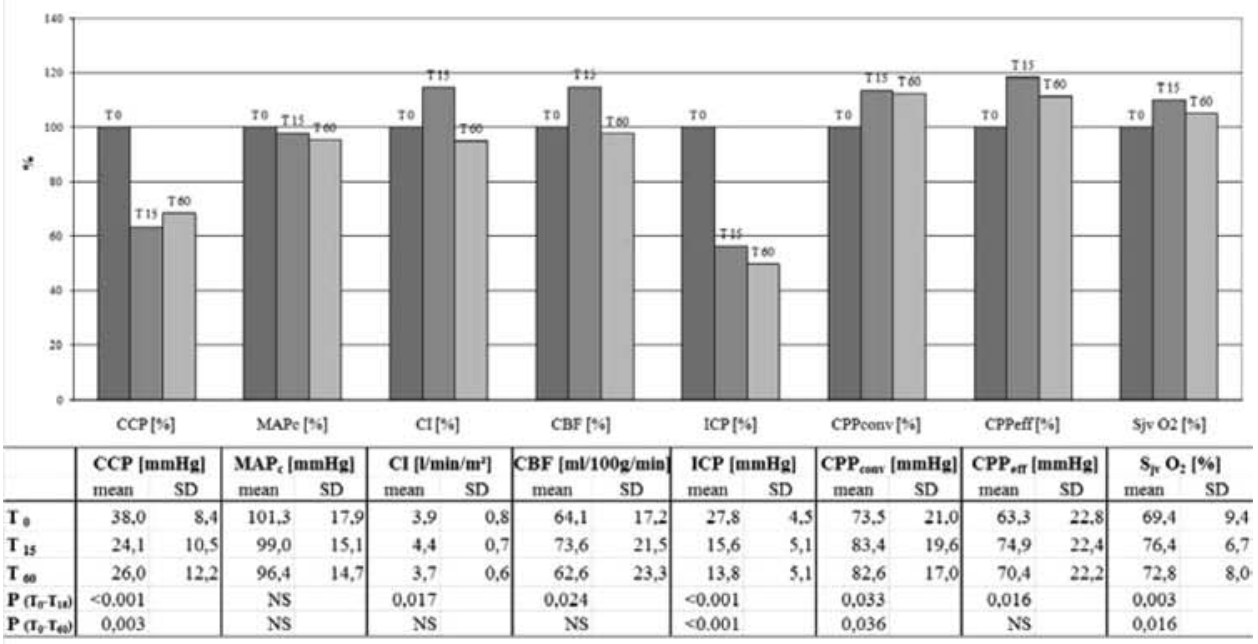

GROUP 2/ MANNITOL

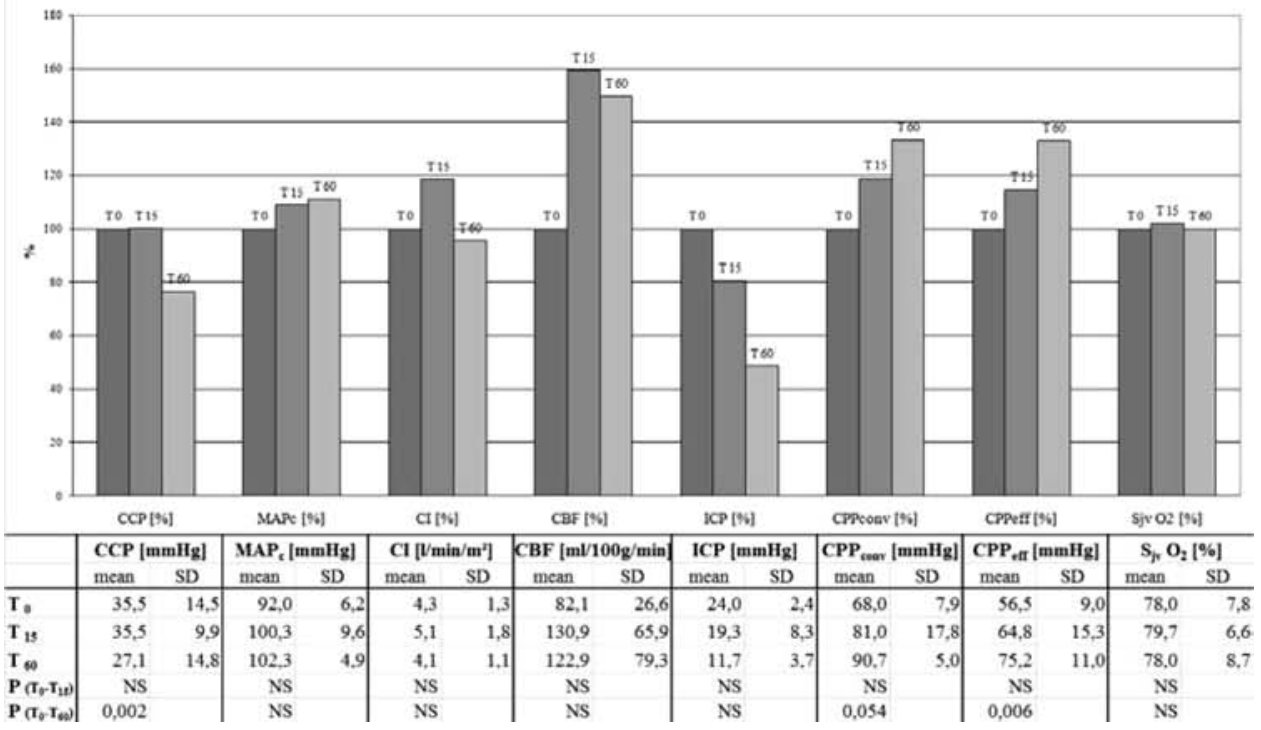


concerning neurointensive care management. Thus, we considered MTBI, TBI, and SAH suitable for evaluation of the two monitoring tools in our study.

\section{TCID Technique}

As compared to intracranial $\mathrm{CBF}$ probes, which measure blood flow in a small region of interest (regional CBF), TCID provides bedside information about global CBF. As a jugular bulb catheter is needed, this method cannot be called non-invasive. However, complications as hematoma, thrombosis, or infection are rare [27]. Indocyanine green has proved to be a safe indicator that does not cause allergic reactions and is not oncogenic [28]. TCID does not allow for continuous monitoring of blood flow, but can be repeated several times per day. The risk of affection of cerebral metabolism by the ice-cold injections is low since jugular bulb temperature changes are usually less than $0.15^{\circ} \mathrm{C}$ [8]. Measurement failures (28.6\% in our series) have been reported previously (34\%) [7], they are easy to detect and hence do not endanger the accuracy of the method. However, as each measurement takes time and a physician, the method demands considerable manpower.

\section{Reliability of Estimation of CCP and $\mathrm{CPP}_{\mathrm{eff}}$}

An essential step to estimate the critical closing pressure (CCP) is the linear extrapolation of a regression line of corresponding values of AP and $V_{\mathrm{MCA}}$ to zero-flow velocity. Legitimation for this extraploration was given by Early et al. [29] who reduced AP to zero flow in the monkey and Aaslid et al. [30] who observed pressure-flow velocity correlation to zero-flow velocity in human beings undergoing a short cardiac arrest for cardioversion. Cerebral autoregulation does not interfere with the calculation, as single-heartbeat-derived pressure changes are too fast for the vascular response [31]. An advantage of $\mathrm{CPP}_{\text {eff }}$ is that it does not, unlike $\mathrm{CPP}_{\text {conv }}$, necessitate placement of an intracranial pressure probe.

\section{Effects of Elevated Ventilation}

$\mathrm{PaCO}_{2}$ induced reduction of cerebral blood volume and hence ICP does not lead to an increase of CBF due to the flow-limiting vasoconstructive response. Our findings are in line with other authors, who observed worsening of CBF under moderate hyperventilation [32-34].

Taking into consideration the elevated vasomotor tone in the small resistance vessels, the resulting perfusion pressure $\mathrm{CPP}_{\text {eff }}$ is physiologically better adapted to real flow conditions. The conventional formula $\mathrm{CPP}_{\text {conv }}=-$ $\mathrm{MAP}_{\mathrm{c}}-\mathrm{ICP}$ does not respect local vessel reactions. According to our findings $\mathrm{CPP}_{\text {conv }}$ cannot be recommended to monitor blood flow conditions in patients undergoing elevated ventilation.

Osmotherapy_Effects, Duration, Complications

Both mannitol and hypertonic saline are standard osmotherapeutic drugs for intracranial hypertension today. The osmolalities of mannitol $20 \%$ and hypertonic saline $7.2 \%$ (Hyperhes ${ }^{\circledR} 7.2 \%$ ) are different, with hypertonic saline $7.2 \%$ being more than twice as strong (2570 mosm/l) as mannitol $20 \%$ (1100 mosm/l). However, equal osmolality is not a methodological demand as the two drugs reduce ICP by partly different pharmacodynamic ways [35, 36]. Hypertonic saline is known to reduce ICP in some cases of mannitolresistant intracranial hypertension [37, 38], whereas mannitol can be applied in patients in whom high sodium serum levels prohibit hypertonic saline administration.

With regard to statistical significance, our findings underline the effectiveness of hypertonic saline concerning reduction of ICP and temporary increase of $\mathrm{CBF}$ as well as the safety of both the drugs (zero adverse effects observed). Furthermore, our findings indicate that mannitol may have stronger and longer lasting effects on CBF than hypertonic saline.

\section{Limitations of the Study}

Regional CBF techniques as intraparenchymal probes give information about regions of interest, e.g., the penumbra. Global CBF cannot provide this information. Conversely, regional $\mathrm{CBF}$ values are unrepresentative for perfusion of the brain as a whole. A simultaneous observation of CPPa global parameter-and $\mathrm{CBF}$ requires a global $\mathrm{CBF}$ technique. TCID presents currently the most handy and repeatable technique of bedside assessment for global CBF. Other methods as Kety-Schmidt or xenon computed tomography, are difficult to apply and limited concerning repeatability. The simultaneous assessment of blood flow and perfusion pressure is important, even if direct correlation between CPP and CBF is methodically incorrect: in clinical practice monitoring at the bedside today is mainly controlled by $\mathrm{CPP}_{\text {conv }}$ - to maintain sufficient cerebral blood flow. However, "real CPP should not be considered a number, but rather a condition for cerebral blood to flow [39]". Therefore, simultaneous assessment of global CBF and of both $\mathrm{CPP}_{\text {conv }}$ and $\mathrm{CPP}_{\text {eff }}$ is important to define how accurately these surrogate parameters indicate blood flow.

TCID is lacking acuity in very low perfusion rates due to its sensitivity to artifact temperature drifts [9, 10]. In normal or elevated cerebral perfusion the method has proven precision. Additionally, important intensive care parameters such as cardiac output, intrathoracic blood volume, and 
lung water simultaneously become available by applying this technique.

$\mathrm{CPP}_{\text {eff }}$ is extrapolated from blood flow velocities in the middle cerebral artery (MCA). In situations of different vasotonus between the two hemispheres the result might not be representative for the contralateral artery. Care has to be taken for the choice of the measured side, e.g., during angiographic vasospasm. Furthermore, $\mathrm{AP}_{\mathrm{c}}$ was measured in the aorta and not in the MCA, which might cause a distortion of the pressure waveform.

The small number of patients with different cerebral pathologies in this study limits statistical analysis for some parameters. Moreover, the two groups were treated with two treatment options (elevated ventilation and osmotherapy) with very different physiological dynamics. The time point measures between the groups were not identical and with the fairly short observation period neither delayed ICP-rebound effects (as known for osmotherapy) nor negative $\mathrm{CBF}$ changes can be precluded after end of surveillance. The aim of our study is not to comment upon the superiority of one treatment over the other. The elaborate assessment was used to obtain simultaneous values of global $\mathrm{CBF}$ and the perfusion pressures $\mathrm{CPP}_{\text {conv }}$ and $\mathrm{CPP}_{\mathrm{eff}}$ during treatment-derived changes in cerebral circulation.

\section{Conclusion}

Both the TCID technique for assessment of global CBF as well as the method of effective CPP proved to be applicable in a neurocritical care setting. Global CBF significantly decreased under elevated ventilation and temporarily increased under osmotherapy. During elevated ventilation, $\mathrm{CPP}_{\text {eff }}$ was superior when compared to conventially obtained CPP in reflecting global CBF changes, while after osmotherapy this correlation was not observed.

Acknowledgment We would like to thank Asita Sarrafzadeh for her very helpful and essential comments.

\section{References}

1. The Brain Trauma Foundation, The Joint Section on Neurotrauma and Critical Care. Guidelines for cerebral perfusion pressure. J Neurotrauma. 2000;17(6-7):507-11.

2. Thees C, Scholz M, Schaller MDC, et al. Relationship between intracranial pressure and critical closing pressure in patients with neurotrauma. Anesthesiology. 2002;96(3):595-9.

3. Burton AC. On the physical equilibrium of small blood vessels. Am J Physiol. 1951;164(2):319-29.

4. Dewey RC, Pieper HP, Hunt WE. Experimental cerebral hemodynamics. Vasomotor tone, critical closing pressure, and vascular bed resistance. J Neurosurg. 1974;41(5):597-606.

5. Permutt S, Riley RL. Hemodynamics of collapsible vessels with tone: the vascular waterfall. J Appl Physiol. 1963;18:924-32.
6. Fegler G. Measurement of cardiac output in anaesthetized animals by a thermodilution method. Q J Exp Physiol Cogn Med Sci. 1954;39(3):153-64.

7. Keller E, Wietasch G, Ringleb P, et al. Bedside monitoring of cerebral blood flow in patients with acute hemispheric stroke. Crit Care Med. 2000;28(2):511-6.

8. Mielck F, Brauer A, Radke O, et al. Changes of jugular venous blood temperature associated with measurements of cerebral blood flow using the transcerebral double-indicator dilution technique. Eur J Anaesthesiol. 2004;21(4):289-95.

9. Mielck F, Wietasch G, Weyland A, et al. Reliability of cerebral blood flow measurements by transcerebral double-indicator dilution technique. Eur J Anaesthesiol. 2001;18(10):653-61.

10. Wietasch GJ, Mielck F, Scholz M, von Spiegel T, Stephan H, Hoeft A. Bedside assessment of cerebral blood flow by doubleindicator dilution technique. Anesthesiology. 2000;92(2):367-75.

11. Marshall L, Marshall S, Klauber M, et al. A new classification of head injury based on computerized tomography. J Neurotrauma 1991;75(Spec. Suppl. Nov):14-20.

12. Servadei F, Murray GD, Penny K, et al. The value of the "worst" computed tomographic scan in clinical studies of moderate and severe head injury. European Brain Injury Consortium. Neurosurgery. 2000;46(1):70-5. (discussion 5-7).

13. Scholz M, Cinatl J, Schadel-Hopfner M, Windolf J. Neutrophils and the blood-brain barrier dysfunction after trauma. Med Res Rev. 2007;27(3):401-16.

14. Ertel W, Keel M, Marty D, et al. Significance of systemic inflammation in 1,278 trauma patients. Unfallchirurg. 1998; 101(7):520-6.

15. Report of World Federation of Neurological Surgeons Committee on a Universal Subarachnoid Hemorrhage Grading Scale. J Neurosurg 1988;68(6):985-6.

16. Lassen N, Peral W. Tracer kinetic methods in medical physiology. New York: Raven Press; 1979.

17. Meier P, Zierler KL. On the theory of the indicator-dilution method for measurement of blood flow and volume. J Appl Physiol. 1954;6(12):731-44.

18. Zierler KL. Theoretical basis of indicator-dilution methods for measuring flow and volume. Circ Res. 1962;10:393-407.

19. Fox IJ, Brooker LG, Heseltine DW, Essex HE, Wood EH. A tricarbocyanine dye for continuous recording of dilution curves in whole blood independent of variations in blood oxygen saturation. Proc Staff Meet Mayo Clin. 1957;32(18):478-84.

20. Herscovitch P, Raichle ME. What is the correct value for the brain-blood partition coefficient for water? J Cereb Blood Flow Metab. 1985;5(1):65-9.

21. Cahill J, Calvert JW, Zhang JH. Mechanisms of early brain injury after subarachnoid hemorrhage. J Cereb Blood Flow Metab. 2006;26(11):1341-53.

22. Kimelberg HK. Current concepts of brain edema. Review of laboratory investigations. J Neurosurg. 1995;83(6):1051-9.

23. Marmarou A, Barzo P, Fatouros P, Yamamoto T, Bullock R, Young $\mathrm{H}$. Traumatic brain swelling in head injured patients: brain edema or vascular engorgement? Acta Neurochir Suppl. 1997;70:68-70.

24. Baethmann A, Kempski OS. Pathophysiology of brain edema. Zentralbl Neurochir. 1997;58(1):20-9.

25. Baethmann A, Jantzen JP, Piek J, Prange H, Unterberg A. Physiology and pathophysiology of intracranial pressure. Zentralbl Neurochir. 1997;58(1):29-31.

26. Sahuquillo J, Poca MA, Arribas M, Garnacho A, Rubio E. Interhemispheric supratentorial intracranial pressure gradients in head-injured patients: are they clinically important? J Neurosurg. 1999;90(1):16-26.

27. Coplin WM, O'Keefe GE, Grady MS, et al. Thrombotic, infectious, and procedural complications of the jugular bulb catheter in 
the intensive care unit. Neurosurgery. 1997;41(1):101-7. (discussion 7-9).

28. Kisch H, Leucht S, Lichtwarck-Aschoff M, Pfeiffer UJ. Accuracy and reproducibility of the measurement of actively circulating blood volume with an integrated fiberoptic monitoring system. Crit Care Med. 1995;23(5):885-93.

29. Early CB, Dewey RC, Pieper HP, Hunt WE. Dynamic pressureflow relationships of brain blood flow in the monkey. J Neurosurg. 1974;41(5):590-6.

30. Aaslid R, Lash SR, Bardy GH, Gild WH, Newell DW. Dynamic pressure-flow velocity relationships in the human cerebral circulation. Stroke. 2003;34(7):1645-9.

31. Barzo P, Bari F, Doczi T, Jancso G, Bodosi M. Significance of the rate of systemic change in blood pressure on the short-term autoregulatory response in normotensive and spontaneously hypertensive rats. Neurosurgery. 1993;32(4):611-8.

32. Muizelaar JP, Marmarou A, Ward JD, et al. Adverse effects of prolonged hyperventilation in patients with severe head injury: a randomized clinical trial. J Neurosurg. 1991;75(5):731-9.

33. Marion DW, Puccio A, Wisniewski SR, et al. Effect of hyperventilation on extracellular concentrations of glutamate, lactate, pyruvate, and local cerebral blood flow in patients with severe traumatic brain injury. Crit Care Med. 2002;30(12):2619-25.

34. Soustiel JF, Mahamid E, Chistyakov A, Shik V, Benenson R, Zaaroor M. Comparison of moderate hyperventilation and mannitol for control of intracranial pressure control in patients with severe traumatic brain injury-a study of cerebral blood flow and metabolism. Acta Neurochir (Wien). 2006;148(8):845-51. (discussion 51).

35. Muizelaar JP, Wei EP, Kontos HA, Becker DP. Mannitol causes compensatory cerebral vasoconstriction and vasodilation in response to blood viscosity changes. J Neurosurg. 1983;59(5): $822-8$.

36. Tseng MY, Al-Rawi PG, Pickard JD, Rasulo FA, Kirkpatrick PJ. Effect of hypertonic saline on cerebral blood flow in poor-grade patients with subarachnoid hemorrhage. Stroke. 2003;34(6): 1389-96.

37. Zornow MH, Todd MM, Moore SS. The acute cerebral effects of changes in plasma osmolality and oncotic pressure. Anesthesiology. 1987;67(6):936-41.

38. Schwarz S, Schwab S, Bertram M, Aschoff A, Hacke W. Effects of hypertonic saline hydroxyethyl starch solution and mannitol in patients with increased intracranial pressure after stroke. Stroke. 1998;29(8):1550-5.

39. Czosnyka M, Matta BF, Smielewski P, Kirkpatrick PJ, Pickard JD. Cerebral perfusion pressure in head-injured patients: a noninvasive assessment using transcranial Doppler ultrasonography. J Neurosurg. 1998;88(5):802-8. 